DOI https://doi.org/10.24297/ijct.v20i.8771

\title{
Dynamic Flight Routing Using Internet of Things Framework
}

\author{
Amlan Chatterjee \\ Department of Computer Science, California State University Dominguez Hills \\ Carson, CA, USA. \\ achatterjee@csudh.edu
}

\begin{abstract}
With increased demand of quicker travel, both for passengers and cargo, there has been major advancements in commercial airlines and number of flights have increased significantly over the last few years. Novel challenges have been introduced due to the rising number of flights in the areas of safety, route planning and maintenance. In addition, for commercial flights, the surge in the number of passengers have also exposed avenues for improving the quality of flight travel, from entering the airport premises to leaving the same at the destination. Although there are many areas of flight and airport operations that can benefit from leveraging technological advancements, choosing safe flight path and making dynamic modifications to it is the critical aspect that needs to be addressed. Comprehending available information to adhere to the provided route and also avoiding known areas of air turbulence, adds to the financial benefit of the commercial airline as well as the safety of the airplane and the passengers are ensured. In this paper, the various aspects of improving the flight routing by providing dynamic intelligent path options to ensure adherence to the provided flight path possible is studied; options for improving the flight safety and turbulence avoidance, which benefits both the passengers and the aircraft are also explored. In addition, keeping aircraft away from conflict zones or war zones, and also from areas of natural disaster, like erupting volcanic ash or forest fires is relevant. Although these issues have been studied before, most of the techniques depend heavily on infrastructure that is on the ground. The basic model requires constant communication with an air traffic control tower, that would provide updates and changes to the flight path as necessary. This leads to some of these methods being unusable on flights operating on oceanic routes and away from the communication zone of the devices placed on land masses. Therefore, in this paper an Internet of Things based framework is proposed to address and handle the above mentioned issues. The framework is structured on the communication model of information exchange among aircraft within the range, as well as taking advantage of ground infrastructure if there is a possible network link to the same. A number of algorithms are proposed for dynamic intelligent routing of flights as well as detection and avoidance of air turbulence. The implementations of the proposed algorithms show improvements ranging from $10 \%$ to $30 \%$ in the methods as compared to using the infrastructure based conventional techniques.
\end{abstract}

Keywords: Internet of Things; IoT; Commercial Aviation; Turbulence Avoidance; Flight path.

\section{Introduction}

Internet of Things (IoT) has become ubiquitous, and technology has evolved significantly with combination of already efficient devices to often add intelligence by communicating with each other. Different types of devices, from daily use gadgets to large scale industrial machines, are now being equipped with a communication component that can send 
and receive data from a plethora of other devices. All of these devices have been operating effectively and performing the specific job they have been designed for. However, rather than operating devices in isolation, an IoT framework can help exchange data that can be analyzed from different perspective and produces hitherto unknown information. IoT based solutions have been applied to many domains to buttress the existing infrastructure and improve the quality of services. While IoT has helped improve cities \& communities, homes, production lines in the industry with their respective smart counterparts, there are many domains of applications that have yet to leverage the benefits of such a framework to build on the existing infrastructure (Jin, Gubbi, Marusic, \& Palaniswami, 2014). Commercial aviation is one such area of industrial application, which can benefit significantly with the use of IoT framework based solutions.

The commercial aviation industry has been growing at a fast pace. There are about 5,000 flights in the airspace of the United States at any given time. With approximately 19,000 operational US airports that operate upwards of 44,000 flights daily, the total number of flights handled by the Federal Aviation Agency (FAA) was more than 16 million in 2019 (United States Department of Transportation 2019). The aviation industry spans across multiple domains of applications. There are various parts that constitute the aviation industry. The major components include line personnel, operations, maintenance, sales \& marketing, reservations \& ticketing, staff personnel, subcontractors and passengers. The line personnel are generally part of engineering \& maintenance, flight operations and sales \& marketing. Operations component of an airline is responsible for the safety and efficiency of aircraft usage. Maintenance ensures all physical components of aircraft is in order. Sales \& marketing together with reservations \& ticketing are the channels of generating revenue by providing services to individual passengers and cargo shipments. Now, all of the different components have issues that can be addressed using technology, specifically IoT based techniques. Solutions have been proposed using IoT based framework to efficiently handle ground operations at airports. Applications also exist to improve passenger service and support as well, including baggage-handling, parking at airports and information about departure and arrivals (Suresh, Kumar, \& Sundararajan, 2015) (Ye-Won \& Yong-Lak, 2015). However, there are very limited applications addressing issues with aircraft during the flight. Flight operations have various different processes; there are extensive procedures for flight path planning, avoiding zones of air turbulence, avoiding no-fly zones, increasing communication with air traffic control towers among others. The above mentioned areas of operations mostly deal with security and safety of the aircraft and the passengers. Therefore, improving any of these operations has high impact in the commercial aviation industry.

Since most of flight operations are well known processes, there have been previous techniques and infrastructure in place to solve these issues. However, with increase in the number of flights over the last few years and the need to improve safety of flights in the wake of recent air disasters, using technology to address the issues is relevant. In this paper, an Internet of Things framework for aircraft is introduced and then the same is leveraged to propose algorithms to improve the safety of flight operations. In particular, we propose algorithms for the following: (a) Maintaining Flight Path, (b) Avoiding different forms of air turbulence, (c) Avoiding no-fly zones and (d) Finding path for flights dynamically. The algorithm for maintaining flight path makes sure the aircraft stays on course for the given route. Avoiding air turbulence is significant in terms of safety of the passengers as well as the structural integrity of the aircraft. Hence, the algorithms propose techniques to avoid the different kinds of air turbulence, like clear air turbulence, mountain wave turbulence, thunderstorm turbulence and wake vortex turbulence. Avoiding no-fly zones, including those that are designated after the flight route is mapped is important. With sudden changes in atmospheric conditions, such as erupting volcanic ashes, flight paths need to be modified dynamically, and these algorithms propose methods for the same. Decreasing the financial cost of operations has major impact on airline operations. Therefore, maintaining optimal flight paths while ensuring safety is required. Since IoT based solutions have certain distinct features that may not be available for technique based on ground infrastructure communication and vice versa, all the proposed algorithms are implemented using simulations and the results are compared to those without using the IoT 
framework.

The outline of the paper is as follows. In Section 2, we provide the literature survey and discuss previous research in the area of commercial aviation with respect to the issues focused on in this paper. We introduce the IoT framework in Section 3. This section discusses in detail the different components of the framework and the patterns of information exchange among the same. The algorithms for different applications of flight operations using the IoT framework is presented in Section 4. The experimental details of the implemented algorithms and corresponding results are discussed in Section 5. Future work, including applications in other domains that can benefit from the algorithms and techniques discussed in this paper, and conclusion is provided in Section 6 .

\section{Related Work}

Although IoT is a relatively novel field of research, there have been previous studies related to various aspects of aviation. Tracking objects based on IoT techniques have been proposed before. However, majority of the research focuses on the real time tracking of physical inventory and other packages, rather than focusing on tracking the aircraft itself (Lee \& Lee, 2015).

In the field of aviation, using IoT based techniques to solve problems have mostly been bound to the domain of safety during the manufacturing process. Current application focus on monitoring aircraft components throughout their manufacturing process to ensure the parts are made correctly (Abdelwahab, Hamdaoui, Guizani, \& Rayes, 2014). There are also methods that deal with monitoring components within the aircraft to confirm appropriate maintenance and function (Internet of Business, 2016).

Tracking aircraft while in flight is one of the important aspects of ensuring safety of the aircraft and the passengers. Therefore, there have been conventional techniques that have existed for a long time for this purpose. Using radar technology is one such method that is widely available. However, there are certain limitations of using radar technology for tracking of flights; these methods do not work in specific areas such as radar shadow zones and also in clutter zones (Sedunov, Sutin, Salloum, Sedunov, \& Masters, 2013).

Since radar based detection systems worked really well in most cases except for the specific areas as mentioned above, there has been research proposed to enhance the usage of radar based techniques to overcome these issues. This has led to advanced technology such as LIDAR (Light Detection and Ranging) systems. Even with these enhancements, there exists a huge potential issue with such techniques; these techniques only work for aircraft that is travelling over land mass or is within a specific range from the nearest land mass (P. Vrancken et al., 2016) (P. S. Vrancken, 2016).

Detecting the location of flights is of utmost importance in commercial aviation. Similarly, there exists other techniques to detect location of other types of vehicles (Da Xu, He, \& Li, 2014) (He, Yan, \& Da Xu, 2014). Detecting location of automobiles is one such problem that has been addressed using IoT framework. Using similar principle, there are other applications in related domains that leverage the features of distributed sensor networks and crowdsourcing (Mohan, Padmanabhan, \& Ramjee, 2008). Creating an Intelligent Transportation System has been proposed before; the basic idea is to provide a tracking application using both automobile-to-automobile and automobile-to-infrastructure (Mallik 2014). The fundamental principle of using sensors on existing devices to track location can be applied to commercial aviation as well, and IoT infrastructure would enable the effective usage of such a scheme.

Methods to predict air turbulence, so that it can be avoided, with certain probability also exist (Sharman, Tebaldi, Wiener, \& Wolff, 2006). However, since commercial aviation incurs heavy damage if passengers or crew are injured, minimizing such incidents using definitive techniques are required. 
There also exists application that perform analysis on airline data using IoT devices. Automatic dependent surveillancebroadcast (ADS-B) systems exchange data between aircraft and receivers contain important location and other related information. These data have been analyzed to be used in applications including airspace and traffic monitoring (Comitz \& Kersch, 2016).

Detecting air turbulence is a well-studied phenomenon, and as a part of it clear air turbulence has been investigated as well. There have been previous research studies that have discussed air turbulence with respect to aviation (Williams. 2014) and specifically the different aspects of clear air turbulence (Barbaresco \& Meier, 2010). Aviation safety from different perspective have been addressed in research using IoT application (Pate \& Adegbija, 2018). There are ways to detect potholes on roads using sensor based applications (Mohan et al., 2008). Although this is based on detecting abnormal conditions using sensors on existing devices, the challenges involved in commercial aviation are fundamentally different.

Our previous work has addressed IoT based techniques for distributed location detection (Chatterjee, Flores, Sen, Hasan, \& Mani, 2019) and also explored Blockchain techniques to enhance data security in communication for aviation (Zakir, Hasan, Wiggins, \& Chatterjee, 2019).

In this paper, scenarios for improving flight operations using both direct and indirect message transfer between nearby aircraft using IoT framework are considered. To the best of our knowledge, this is the first work that proposes using IoT based techniques to detect location of aircraft and dynamically propose changing the route depending on varying conditions.

\section{IoT Framework}

With advancements in aviation technology, aircraft are now equipped with multiple devices of various capabilities. Also, for the operation of the aircraft there are numerous sensors employed as well. Considering an aerial region, with the growth in the commercial aviation sector resulting in the huge increase in the number of flights operating between various geographical locations, there are usually multiple aircraft present within the specific region. Hence, these devices which can both gather and exchange data, form the basis of the IoT framework for aviation. In this Section, an IoT framework that can be used for the aircraft to solve and improve various operational issues is discussed in detail.

All aircraft are equipped with a number of devices to maintain proper operations. Aviation electronics or avionics typically include devices for communication, navigation, control, monitoring, weather and anti-collision systems. Navigation is done using a combination of satellite-based (GPS) and ground-based systems. For the collision avoidance, there are a multitude of systems available; traffic alert and collision avoidance systems (TCAS) helps detect nearby aircraft by actively exchanging data with transponders of nearby aircraft. Aircraft are also equipped with groundproximity awareness systems (GPWS), which uses radar altimeters, and terrain awareness warning system (TAWS). The Aircraft Data Network (ADN) specification proposes data networking standards for use in commercial aircraft installations. The standard provides methods to adapt commercial off-the-shelf (COTS) networking standards to aviation environment.

Therefore, the IoT framework consists of sets of two major components, the air traffic control (ATC) tower and aircraft. The set of ATC towers can be denoted by $\{T\}$ and the set of aircraft denoted by $\{A\}$, so that the total components 


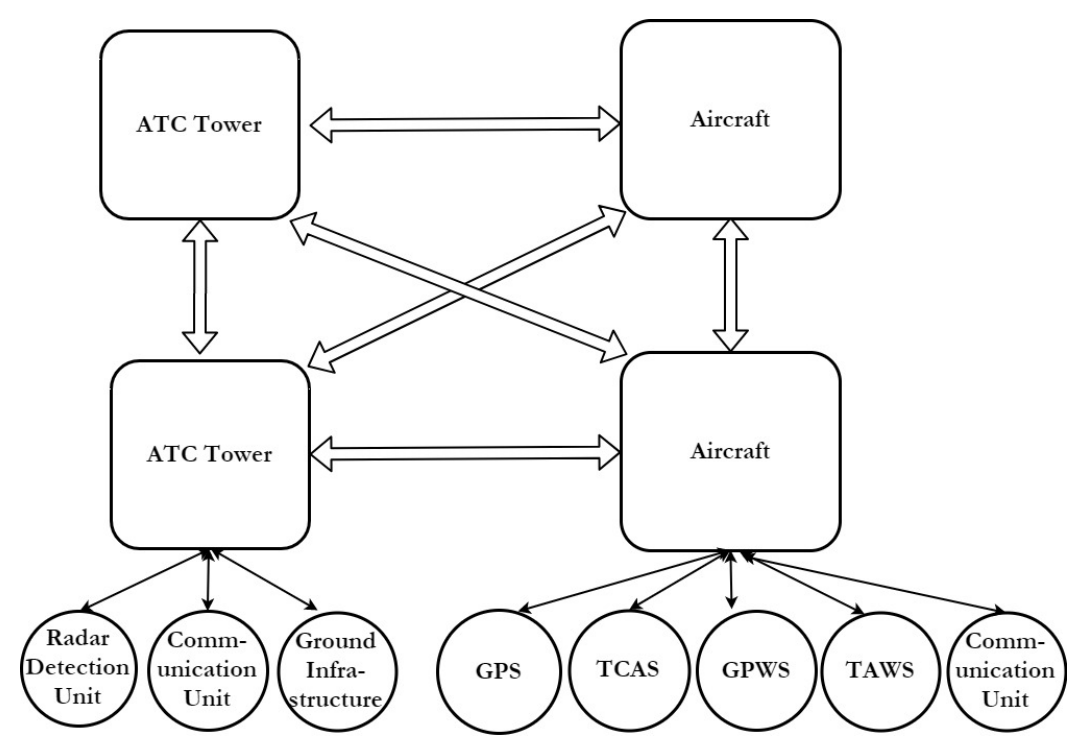

Figure 1: IoT Framework for Aviation

in the system $\{S\}$ is given by

$$
\{S\} \leftarrow\{T \mid T \in \mathbb{N}\} \cup\{A \mid A \in \mathbb{N}\}
$$

where $\mathbb{N}$ is the set of Natural Numbers. The proposed framework is shown in Fig. 1. As evident from the framework, messages can be exchanged between the different elements of the sets of the components. Data in the form of messages can be exchanged between the entities. The messages are denoted using the source and destination information as given by

$$
M_{a, b} \Leftarrow\left\{M_{a} \rightarrow M_{b}\right\}, a \in\{S\}, b \in\{S\},
$$

where $M$ is the message data. An example of the components of the framework in action is given in the Fig. 2
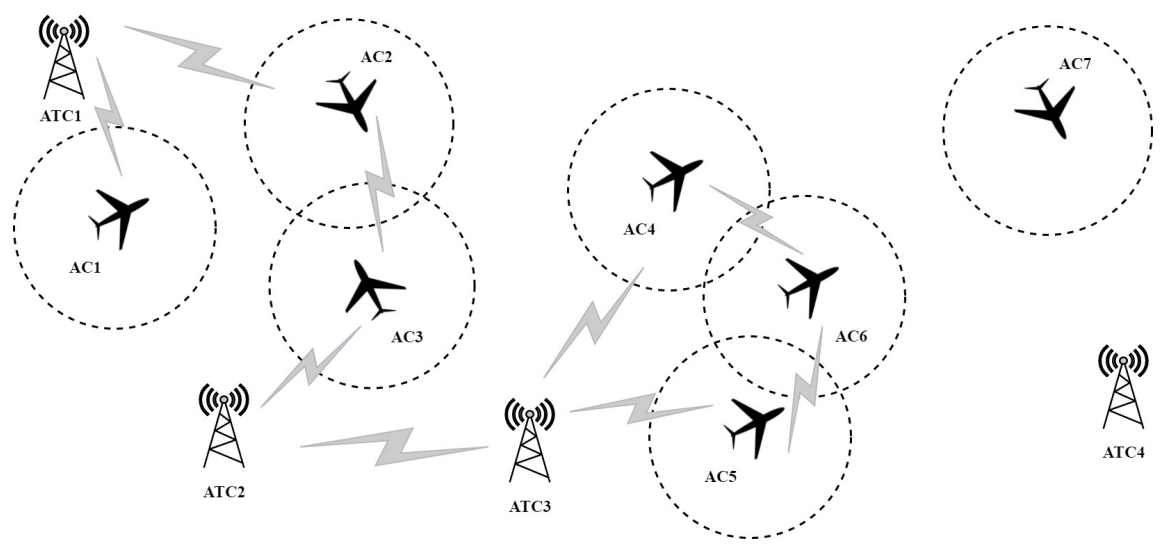

Figure 2: Components of Framework in Action

In the scenario depicted in Fig. 2, elements of both the components are present. For this specific case,

$$
\{T\}=\{A T C 1, A T C 2, A T C 3, A T C 4\}
$$


and, for the aircraft is given by

$$
\{A\}=\{A C 1, A C 2, A C 3, A C 4, A C 5, A C 6, A C 7\} .
$$

So, there are 4 ATC towers and 7 aircraft in the region that is considered in Fig. 2 There are communication links available between aircraft and ATC towers and also direct contact between aircraft the are being tracked by different ATC towers. Also, there are elements from both set of components that are isolated and not in range of any other elements. In the given scenario, $A C 7$ and $A T C 4$ are operating in isolation. In addition, there are elements of the components that are not in direct communication range, but can exchange information via other elements. For example, towers $A T C 1$ and $A T C 2$ cannot communicate directly. However, using the aircraft $A C 2$ and $A C 3$ the ATC towers can exchange information. Therefore, using the proposed framework, data can be exchanged directly among aircraft and ATC towers, and also via each other.

Considering the IoT framework, a generic region could have multiple aircraft within the airspace and multiple air traffic control towers present. It can be assumed that all aircraft within a region can communicate with other aircraft within the same region provided the communication device has no hardware or software malfunction. Now, presence of ATC towers do not guarantee those to be in range of all aircraft. The ATC towers might be in direct communication with only a sub-set of the aircraft in the region. Hence, in the proposed IoT framework there can be different scenarios for the arrangement of the aircraft and the ATCs, as given by the following.

1. Single aircraft, not in range of any ATC: In this scenario, the precise location of the aircraft cannot be determined either by radar based techniques or by using IoT based methods. Since there is a single entity in the region, the IoT framework is not able to assist without the provision for exchange of data due to the lack of other devices. However, in practice this case is rare considering the number of aircraft that is airborne at any instant of time, and given that most aircraft travel on similar routes.

2. Single aircraft, in range of single ATC: This is a basic case scenario. In this case, the aircraft under consideration is in direct contact of the ATC. Therefore, using conventional radar based techniques, the precise location of the aircraft can be tracked at all times during the flight. The IoT based framework in this case consists of two entities: the aircraft and the ATC tower. Based on the communication model, where there would be data exchanges between the aircraft and the ATC tower, the precise location of the aircraft will be detected at all times using the IoT system as well.

3. Single aircraft, in range of multiple ATCs: In this case, the aircraft is in direct contact with multiple ATCs, which might or might not be in direct contact with each other. The IoT framework would consist of single aircraft and multiple ATCs. The precise location of the aircraft can be tracked by all the ATCs in the region. In addition, there might be options for the aircraft to act as an intermediary to exchange information among the ATCs that are not in direct contact with each other. This in turn can provide additional information to ATCs to get location data of a larger region.

4. Multiple aircraft, none in range of any ATC: In this case, the IoT framework would consist of just aircraft. The precise location of the aircraft cannot be tracked by any ATC. However, using triangulation principle, approximate location of aircraft can be calculated, based on last known location, and the information shared among the aircraft.

5. Multiple aircraft, subset in range of single ATC: In this case, with some of the aircraft in range of the ATC provides the idea scenario for using the IoT framework to track an aircraft that is not within range of ATC 
via information exchanged through other aircraft. This forms the backbone of the argument to have an IoT framework for aircraft in the first place. The aircraft that are within the range of the ATC can exchange the location data directly. The aircraft that are not in direct communication with the ATC can still exchange data using multiple hops.

6. Multiple aircraft, all in range of single ATC: In this case, all aircraft can be precisely tracked by the ATC. Aircraft can communicate directly with the ATC and the presence or absence of IoT framework have no influence on the tracking percentages.

7. Multiple aircraft, subset in range of multiple ATCs: In this case, the subset of the aircraft that are in direct contact with the ATC can exchange the location data directly. For the other aircraft, the data exchange happens via the subset of aircraft which are in direct contact. So, in this case, the precise location of all aircraft can be determined using algorithms that can use triangulation on aircraft to determine the exact location using other aircraft that are in direct contact. Since there are multiple ATCs involved, data exchange between the ATCs is also possible via the aircraft.

8. Multiple aircraft, all in range of multiple ATCs: In this case, the precise location of all aircraft can be tracked by all the ATCs. In addition, if the ATCs are not in direct contact with each other, then information can be exchanged using the aircraft for forwarding and sending the messages. Such scenarios with multiple groups of aircraft and intersecting groups of ATCs can exchange enough information to form a network where all aircraft and ATCs have precise location information of all other aircraft within as well as outside of the region.

\section{Dynamic flight routing algorithms}

The current techniques to detect the location of an aircraft and communicate corrective measures depend on radar based techniques. As mentioned earlier, the radar based techniques do not work when the aircraft is in radar shadow zones, or radar clutter zones and also when entities are out of range. For aircraft where the entire flight path is over land mass, these issues are non existent because of the coverage of radar and the presence of multiple radars. However, these techniques are not reliable when the aircraft is outside of radar zone for a significant portion of the flight path. Such a scenario, where the aircraft is out of the range of radar, is common for aircraft on flight paths over oceans. In this case, using the IoT framework for aircraft can be used to determine location of aircraft and take corrective measures. Therefore, in this Section, algorithms to address the following issues are introduced (a) Maintaining Flight Path, (b) Avoiding different forms of air turbulence, (c) Avoiding no-fly zones and (d) Finding path for flights dynamically.

\subsection{Maintaining Flight Path:}

Flight paths are calculated in entirety from the origin to the destination for commercial flights. However, due to various reasons, deviation from the planned flight path is required. Once a deviation is undertaken, the flight path must be recalculated and communicated to the aircraft under consideration. Usually, the parameters for choosing a specific flight path depends on different characteristics such as shortest distance, avoiding areas of air turbulence, and also avoiding no-fly zones. Therefore, to avoid any disruptions, the flight path is provided to the aircraft and it is supposed to follow the same. If there is any deviation from the path, the proposed IoT framework would provide corrective measure. If the aircraft is in the range of an air traffic control (ATC) tower, directions to get back on the 
planned path can be provided directly. In case the aircraft can communicate with an ATC tower based on the IoT framework via other aircraft, the corrective measure can be provided over the framework indirectly. The technique for maintaining the flight path is given in Algorithm 1

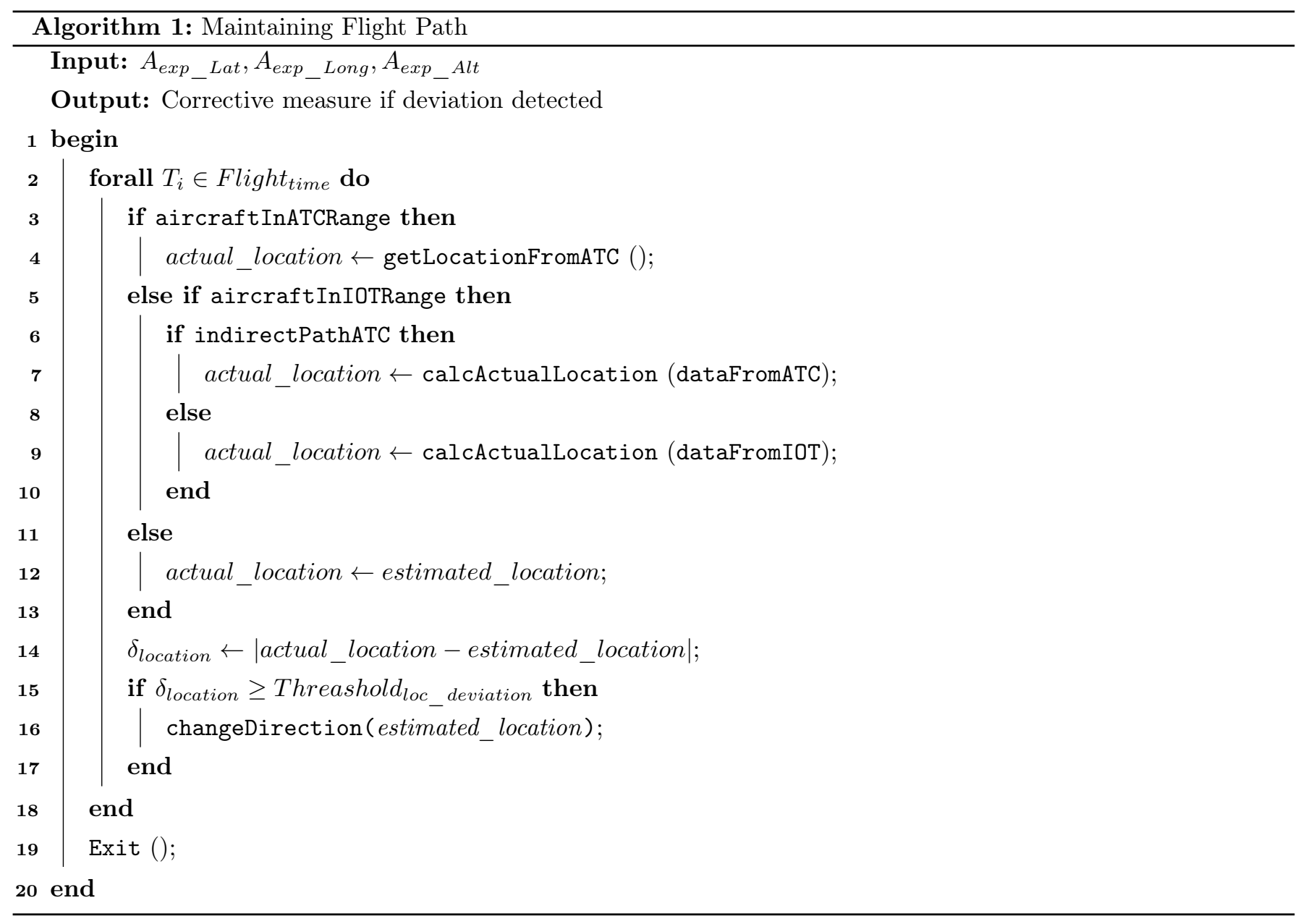

\subsection{Avoiding different forms of air turbulence:}

Avoiding any form of turbulence is beneficial for the safety of both the passengers and the aircraft. There are various forms of air turbulence that can be encountered by an aircraft. The common types of turbulence include clear air turbulence, thermal turbulence, temperature inversion turbulence, mechanical turbulence, frontal turbulence, mountain wave turbulence, thunderstorm turbulence and wake vortex turbulence. Now, broadly classifying, the thermal, temperature inversion, mechanical and frontal turbulence all belong to the major category of clear air turbulence. Hence, there are mainly four broad categories of turbulence: clear air turbulence, mountain wave turbulence, thunderstorm turbulence and wake vortex turbulence. In this sub-section, methods for avoiding these types of turbulence using the IoT framework is discussed.

\subsubsection{Avoiding clear air turbulence:}

Clear air turbulence (CAT) is one of the most difficult turbulences to avoid. There are techniques that exist which can be used to predict the potential location of CATs based on thermal and other atmospheric conditions, but still it 
cannot be completely avoided. The main idea here is to minimize the effects of clear air turbulence in a specific zone by changing flight route once a CAT is detected by an aircraft within the region.

\subsubsection{Avoiding mountain wave turbulence:}

Due to the existence of rugged terrain and the flow of winds along the sides of mountains, there exists mountain wave turbulence. The effect of mountain wave turbulence can extend over large gerographical areas, and also far beyond the actual mountainous region. Using the IoT framework, the different devices that exist on aircraft can be used to measure the changes in the atmospheric condition that can lead to such turbulence. Hence, once an aircraft detects the potential presence of such a disturbance, the flight path of that aircraft and all other aircraft in the region is required to be updated to avoid the zone.

\subsubsection{Avoiding thunderstorm turbulence:}

Thunderstorms usually range over a large area and has the potential to have turbulence spread across the region. Due to the size of the area affected, there might be more aircraft involved in thunderstorm turbulence as compared to the other forms of air turbulence. Hence, there is a potential of false positives while providing alert to aircraft in the region.

\subsubsection{Avoiding wake vortex turbulence:}

Wake vortex turbulence is a special type of clear air turbulence. This type of turbulence is caused by the huge displacement of air caused by a moving aircraft. If the speed and the position of the last aircraft to travel through the region is known, then the effect of the aircraft on the wind force for the region can be calculated. Considering the IoT framework, the different aircraft can communicate among each other and provide the specific details that can help identify the region and avoid the space for a given time for the effect to be nullified.

\subsection{Avoiding no-fly zones}

Although the flight path that is provided to aircraft avoids all the no-fly zones, there might be dynamic changes to the route options that needs to be addressed. In case of flight deviations from the proposed flight path, there are algorithms proposed in the previous sections of the paper that can assist in getting the flight back on track. However, there might be situations where due to change in atmospheric conditions, certain areas become designated no-fly zones after the current flight path is generated. One example of such a condition can be the eruption of volcanic ash that shoots up high in the air and can cause the sensors on aircraft to malfunction. Another example can be rapidly spreading forest fires, that tend to produce lot of smoke and toxic materials, and it is better to avoid flying of aircraft over such regions. In addition, conflict areas and war zones also need to be avoided in case of escalating tension, whereby commercial aircraft may be targeted. In such cases, the area of space above the specific zone is designated a no-fly zone. Aircraft that are still in direct contact of ATC towers can get the information as well as the updated flight path directly from the same. For aircraft that is outside the range of any ATC tower, using the IoT framework, information about the no-fly zone can be communicated to the specific aircraft via other aircraft. Also, as required, the new flight path co-ordinates can be supplied indirectly. 


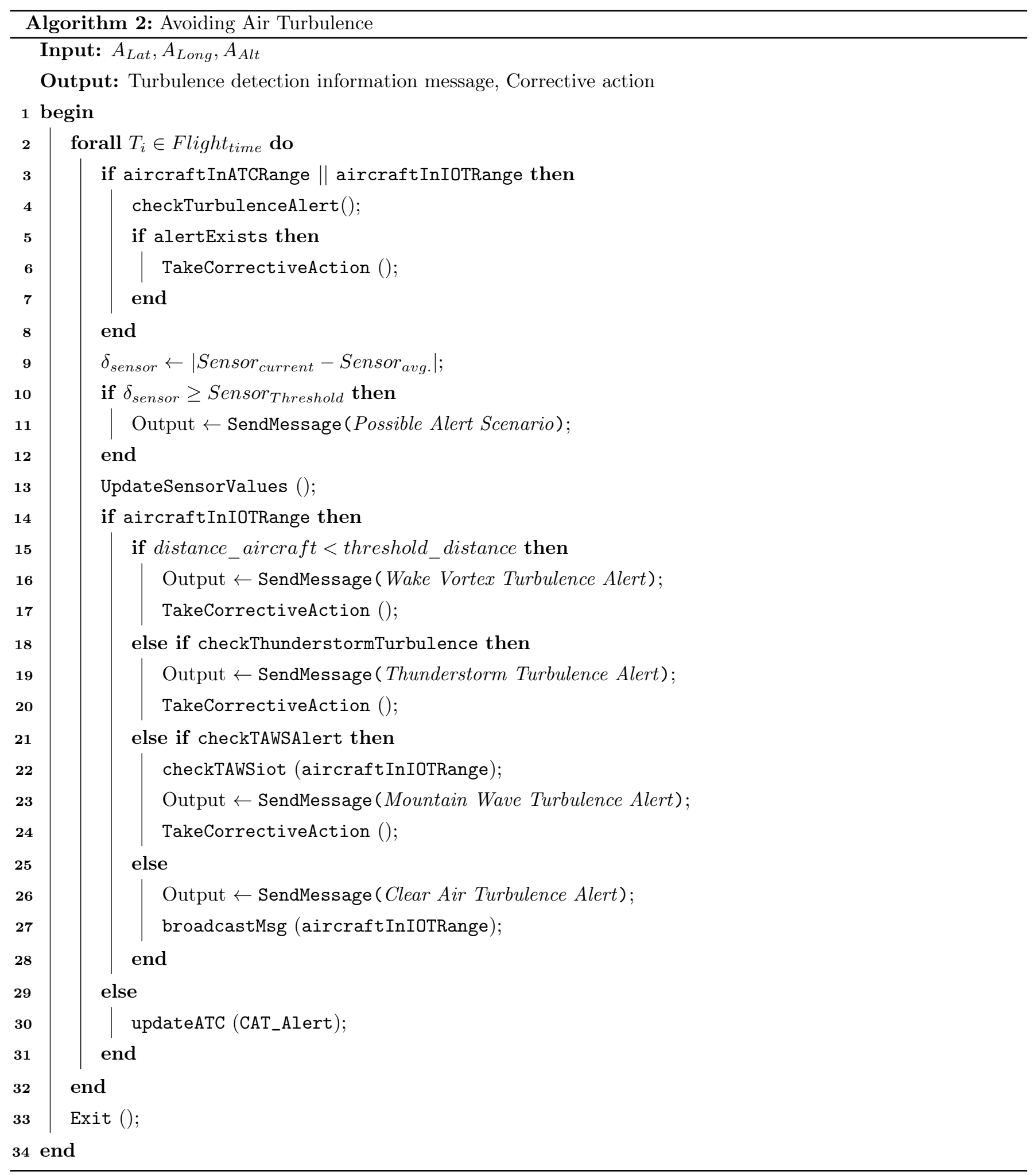

\subsection{Dynamic flight path calculations}

The flight path is decided based on multitude of factors. Once the flight path is communicated to the aircraft, the route is followed and deviations are avoided. However, due to changing weather conditions or other factors, modifications to the flight path might be required. In this case, the remaining flight path needs to be calculated and communicated 




to the aircraft. If the aircraft is within the range of an ATC, then the calculations can be done by the ATC and the path directly communicated to the aircraft. If the the aircraft is outside the range of any ATC, request for updated flight path can be made via other aircraft to the ATC; then the updated flight path is communicated back using the IoT framework involving multiple hops. In the special case, when the aircraft under consideration is outside the range of any ATC and so are the other aircraft in the region with no connection available to any ATC, the aircraft can exchange information among themselves, and then the new path might be as simple as copying another flights path with minimum delay. For the sake of safety, the new flight path must be checked once a communication link with an ATC is available.

\section{$5 \quad$ Experimental Results}

For the domain of commercial aviation, different scenarios relevant to aircraft safety and proper operations have been considered in this paper. Multiple algorithms have been proposed to solve issues with each of the scenarios under consideration. All the algorithms are implemented using simulation techniques and the results are discussed in this Section.

For maintaining the flight path, as described in Algorithm 1, the percentage of the flights that can benefit from the proposed method is compared with that of traditional radar based technology. The implementation for all the algorithms assume flights operating over oceanic routes, since this is the challenging area for the current scenarios in commercial aviation. In the simulation, the number of flights are increased gradually over time from 1 to 300 , and the percentage of flights that maintained the path is reported. From the result, as given in Fig. 3 , it is evident that the 

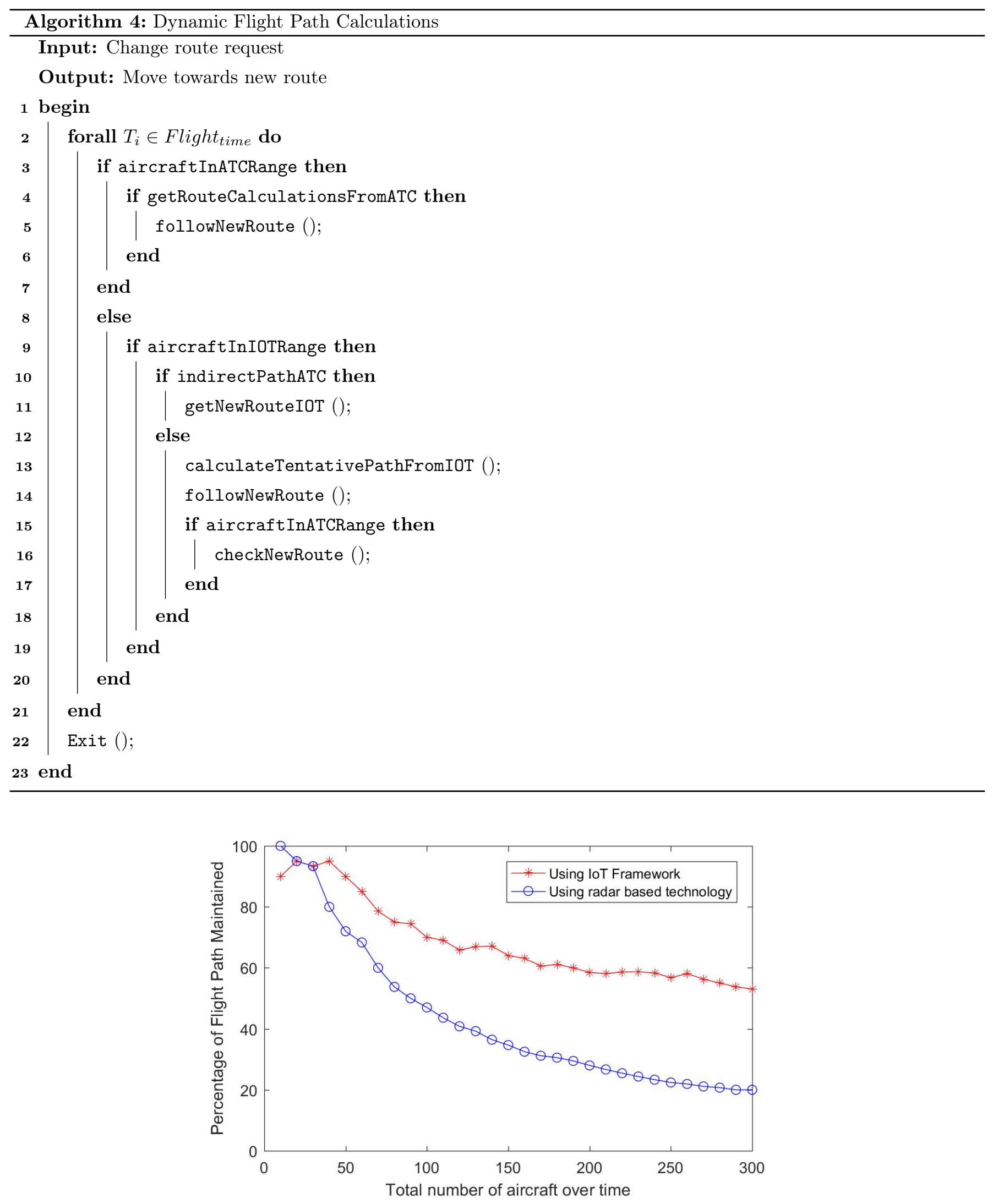

Figure 3: Maintaining Flight Path 
IoT based technique performs better than the radar based technique. In the initial part, the performance of the radar based technique is comparable to the IoT based technique, since at that time most of the flights that have departed from the source are still in contact with the ATC tower. With more flights moving away from the towers over time, the performance of the IoT technique is better. On average, the proposed method maintains the path for $68 \%$ of flights as compared to the traditional method for $42 \%$.



Figure 4: Detecting Air Turbulence

The algorithm for avoiding air turbulence, as given in Algorithm 2, is also implemented, and the results are reported in Fig. 4. In the implementation, a total of 300 flights are considered, and over time, the percentage of flights experiencing turbulence is varied from $1 \%$ to $20 \%$ of the total flights. Turbulence alert warnings to nearby aircraft is reported as a percentage for both the IoT based and radar based techniques. The IoT based technique performs better with the increase in the number of aircraft. On an average, the proposed method is able to provide warnings to $48 \%$ of the aircraft in the region as compared to $20 \%$ for the radar based technique. However, since the IoT based technique does not take into account the direction of other aircraft, as to whether those are headed towards the turbulence or away from it, there is a potential for false alerts with respect to an aircraft. The percentage of false alerts are also reported; on an average there are $5 \%$ of false alerts possible using the proposed method.

Fig. 5 presents the implementation of the algorithm to avoid no-fly zones, as given in Algorithm 3 . Usually flights do not deviate often, so for the simulation, the percent of flights deviating are varied over time from $1 \%$ to $20 \%$ percent of the total number of flights. Leveraging the advantages of the proposed IoT framework, the introduced technique performs better for majority of the cases where there are enough airborne aircraft. On an average, the proposed method provides avoidance of no-fly zone information to aircraft in $54 \%$ of the cases as compared to $22 \%$ in the conventional technique.

The algorithm for dynamic path calculations, as described in Algorithm 4 is implemented and the results provided in Fig. 6. The proposed IoT based methods performs better than conventional radar based technology. The percentage of aircraft that can communicate with an ATC tower via other aircraft using the IoT framework is quite high, with an average of $70 \%$ of aircraft in range of other aircraft. In case of no path from the aircraft to any ATC tower, the algorithm proposes utilizing paths of aircraft following same route with some restrictions. Using the IoT based method in isolation from any ATC tower, the average percent of aircraft that can benefit from the technique is $48 \%$, as compared to $38 \%$ using infrastructure based techniques. 


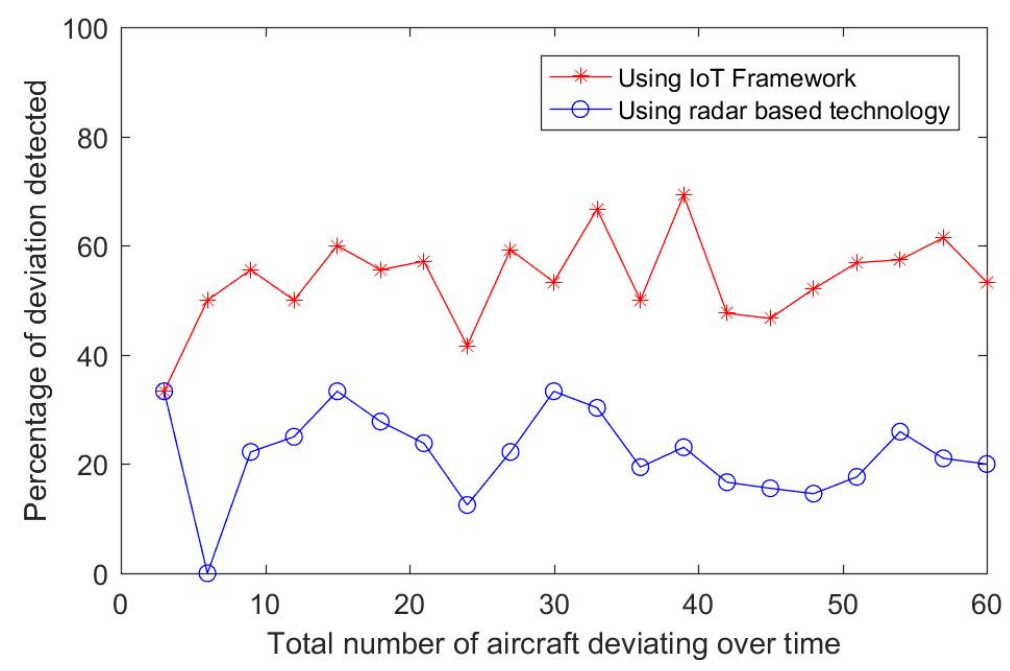

Figure 5: Avoiding No-Fly Zone

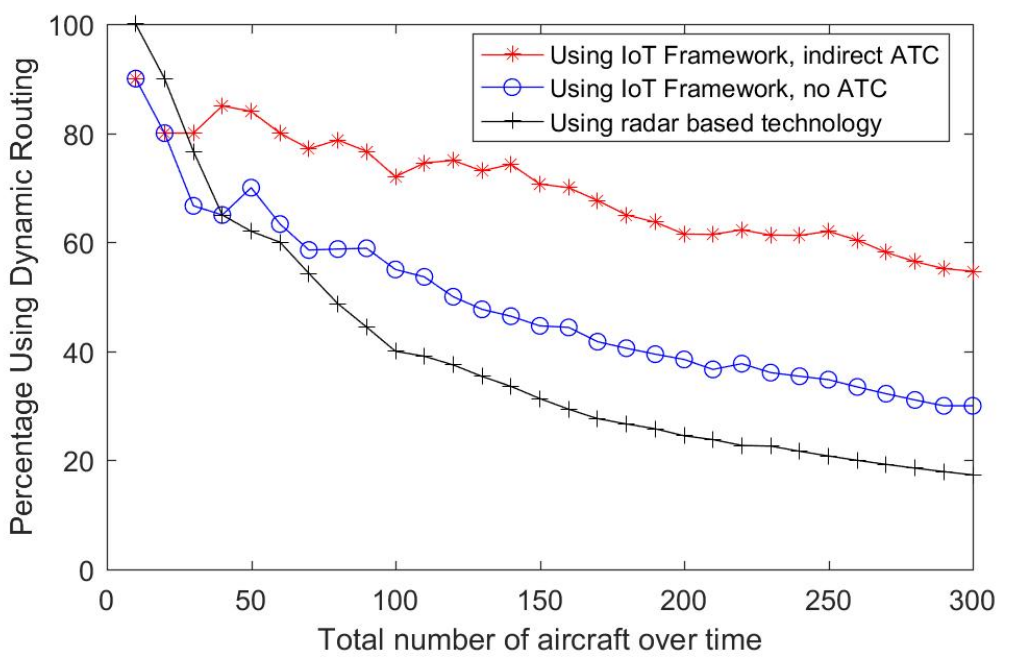

Figure 6: Dynamic Flight Path Calculation

\section{Conclusion}

In this paper, different aspects of commercial aviation that can leverage technological advancements are studied. Algorithms are provided to address issues related to providing dynamic safe flight routing path and avoiding turbulence using a proposed IoT framework. The basis of the IoT framework and the proposed algorithms take into consideration the capability of aircraft that are equipped with multiple sensing and communicating devices to exchange information. Along with the ground infrastructure based radar sensing technology and communication with air traffic control towers that provide assistance to flights over land masses, the methods discussed in this paper provide novel solutions for the challenges in commercial aviation with aircraft to aircraft communication that are effective even on oceanic routes. The proposed algorithms are compared with conventional techniques, and it is evident from the experimental results that the IoT based solutions are efficient than the existing solutions. The results show that introducing IoT based solutions to the critical areas of commercial aviation can be significantly beneficial. As part of future work, the same principles and algorithmic solutions would be applied to other related domains to solve problems specific to the same. 


\section{References}

Abdelwahab, S., Hamdaoui, B., Guizani, M., \& Rayes, A. (2014). Enabling smart cloud services through remote sensing: An internet of everything enabler. IEEE Internet of Things Journal, 1(3), 276-288. doi: https:// doi.org/10.1109/JIOT.2014.2325071

Barbaresco, F., \& Meier, U. (2010). Radar monitoring of a wake vortex: Electromagnetic reflection of wake turbulence in clear air. Comptes rendus physique, 11(1), 54-67. doi: https://doi.org/10.1016/j.crhy.2010.01.001

Chatterjee, A., Flores, H., Sen, S., Hasan, K. S., \& Mani, A. (2019). IoT-based algorithms for distributed location detection for flights. International Journal of Hybrid Intelligence, 1 (2-3), 191-210. doi: https://doi.org/10.1504/ IJHI.2019.103578

Comitz, P., \& Kersch, A. (2016). Aviation analytics and the internet of things. In Integrated communications navigation and surveillance (icns), 2016 (pp. 2A1-1). doi: https://doi.org/10.1109/ICNSURV.2016.7486327

Da Xu, L., He, W., \& Li, S. (2014). Internet of things in industries: A survey. IEEE Transactions on industrial informatics, 10(4), 2233-2243. doi: https://doi.org/10.1109/TII.2014.2300753

He, W., Yan, G., \& Da Xu, L. (2014). Developing vehicular data cloud services in the iot environment. IEEE Transactions on Industrial Informatics, 10 (2), 1587-1595. doi: https://doi.org/10.1109/TII.2014.2299233

Internet of Business. (2016). 10 stellar real-life examples of IoT taking flight in aviation. https://internetofbusiness.com/10-real-life-examples-iot-aviation/.

Jin, J., Gubbi, J., Marusic, S., \& Palaniswami, M. (2014). An information framework for creating a smart city through internet of things. IEEE Internet of Things Journal, 1(2), 112-121. doi: https://doi.org/10.1109/ JIOT.2013.2296516

Lee, I., \& Lee, K. (2015). The Internet of Things (IoT): Applications, investments, and challenges for enterprises. Business Horizons, 58(4), 431-440. doi: https://doi.org/10.1016/j.bushor.2015.03.008

Mallik, S. (2014). Intelligent transportation system. International Journal of Civil Engineering Research, 5(4), 367-372.

Mohan, P., Padmanabhan, V. N., \& Ramjee, R. (2008). Nericell: rich monitoring of road and traffic conditions using mobile smartphones. In Proceedings of the 6th acm conference on embedded network sensor systems (pp. 323-336). doi: https://doi.org/10.1145/1460412.1460450

Pate, J., \& Adegbija, T. (2018). Amelia: An application of the internet of things for aviation safety. In 2018 15th IEEE Annual Consumer Communications $\&$ Networking Conference (CCNC) (pp. 1-6). doi: https://doi.org/ 10.1109/CCNC.2018.8319163

Sedunov, A., Sutin, A., Salloum, H., Sedunov, N., \& Masters, D. (2013). Passive acoustic localization of small aircraft. In Proceedings of Meetings on Acoustics 166ASA (Vol. 20, p. 055005). doi: https://doi.org/10.1121/1.4830889

Sharman, R., Tebaldi, C., Wiener, G., \& Wolff, J. (2006). An integrated approach to mid-and upper-level turbulence forecasting. Weather and forecasting, 21(3), 268-287. doi: https://doi.org/10.1175/WAF924.1

Suresh, M., Kumar, P. S., \& Sundararajan, T. (2015). IoT based airport parking system. In Innovations in information, embedded and communication systems (iciiecs), 2015 international conference on (pp. 1-5). doi: https://doi.org/ 10.1109/ICIIECS.2015.7193216

United States Department of Transportation.

(2019).

Federal Aviation Agency. https://www.faa.gov/air_traffic/by_the_numbers/.

Vrancken, P., Wirth, M., Ehret, G., Barny, H., Rondeau, P., \& Veerman, H. (2016). Airborne forward-pointing UV Rayleigh lidar for remote clear air turbulence detection: system design and performance. Appl. Opt., 55(32), 9314-9328. Retrieved from http://ao.osa.org/abstract.cfm?URI=ao-55-32-9314 doi: 10.1364/ AO.55.009314 
Vrancken, P. S. (2016). Airborne Remote Detection of Turbulence with Forward-Pointing LIDAR. In R. Sharman \& T. Lane (Eds.), Aviation turbulence: Processes, detection, prediction (pp. 443-464). Cham: Springer International Publishing. Retrieved from https://doi.org/10.1007/978-3-319-23630-8_22 doi: 10.1007/ 978-3-319-23630-8_22

Williams, J. K. (2014). Using random forests to diagnose aviation turbulence. Machine Learning, 95(1), 51-70. Retrieved from https://doi.org/10.1007/s10994-013-5346-7 doi: 10.1007/s10994-013-5346-7

Ye-Won, L., \& Yong-Lak, C. (2015). Proposal for Air-Baggage Tracing System Based on IoT. In 2015 9th international conference on future generation communication and networking (fgcn) (p. 25-28). doi: 10.1109/FGCN.2015.15

Zakir, Y., Hasan, K. S., Wiggins, N. S., \& Chatterjee, A. (2019). Improving Data Security in Message Communication between ACT and Aircraft using Private Blockchain. In 2019 Sixth International Conference on Internet of Things: Systems, Management and Security (IOTSMS) (pp. 506-513). doi: https://doi.org/10.1109/ IOTSMS48152.2019.8939209 